New modular form identities associated to generalized vertex operator algebras

\author{
Alexander Zuevsky
}




\title{
NEW MODULAR FORM IDENTITIES ASSOCIATED TO GENERALIZED VERTEX OPERATOR ALGEBRAS
}

\author{
ALEXANDER ZUEVSKY
}

Received 21 February, 2014

\begin{abstract}
New identities appearing from consideration of higher genus characters for generalized vertex operator algebras with a formal VOSA parameter associated to a local coordinate on a self-sewn Riemann surface are considered. Genus two version of twisted product Jacobi identity is reviewed. Further generalization of classical number theory identities for modular forms are proposed.
\end{abstract}

2010 Mathematics Subject Classification: 17B69; 30F10; 32A25; 11F03

Keywords: vertex operator superalgebras, intertwining operators, Riemann surfaces, automorphic forms, theta-functions, Jacobi product identities

\section{INTRODUCTION}

This is the a continuation of the papers [42,43] describing modular form identities obtained as a result of computation of characters for vertex operator algebra modules on genus two (and higher) Riemann surfaces [33-38]. In thise part we announce new modular form identities proved (in collaboration with M.P. Tuite) in [37] and obtained in the consideration of genus one and genus two characters for generalized vertex operator algebras with a formal VOSA parameter associated to a local coordinate on a self-sewn Riemann surface.

In $[28,34,37]$ we have established modular properties of genus one and genus two partition and $n$-point functions for free fermionic vertex operator superalgebras. Modular properties of correlations functions for classes of vertex algebras on the torus were proven in [7,29,41], [21-28], [33-38] and others. In [34] we established that the genus two partition function for the rank two free fermion VOSA is modular invariant with respect to the group $G=(S L(2, \mathbb{Z}) \times S L(2, \mathbb{Z})) \rtimes \mathbb{Z}_{2}$, i.e.,

$$
Z_{V_{\mathbb{Z}}}^{(2)}\left[\begin{array}{l}
f^{(2)} \\
g^{(2)}
\end{array}\right] \mid \gamma=e_{\gamma}^{(2)}\left[\begin{array}{l}
f^{(2)} \\
g^{(2)}
\end{array}\right] Z_{V_{\mathbb{Z}}^{(2)}}\left[\begin{array}{l}
f^{(2)} \\
g^{(2)}
\end{array}\right],
$$

where $\mid \gamma$ denotes $G$-action on the partition function, and $e_{\gamma}^{(2)}\left[\begin{array}{l}f^{(2)} \\ g^{(2)}\end{array}\right]$ is a multiplier system. Note that modular invariance of the genus two partition function can also 
inferred from using modular properties of the Riemann theta function and the Heisenberg genus two partition function described in [21]. Similarly, we can also obtain $[34,37]$ modular invariance for the two rank two free fermion $n$-point generating differentials $\mathscr{E}_{V_{\mathbb{Z}}, n}^{(2)}\left[\begin{array}{l}f^{(2)} \\ g^{(2)}\end{array}\right]\left(x_{1}, y_{1}, \ldots, x_{n}, y_{n}\right),(5.4)$ as well as for all $n$-point function differentials $\mathcal{F}_{V_{\mathbb{Z}}, n}^{(2)}\left[\begin{array}{l}f^{(2)} \\ g^{(2)}\end{array}\right]\left(\left(v_{1}, z_{1}\right), \ldots,\left(v_{n}, z_{n}\right)\right)$ (5.3) (see subsection 5.2 below) and extend this result to higher genus [34]. In more complicated cases at genus $g$ [38] we can explicitly prove automorphic properties of generating functions by using their explicit representations following from vertex operator algebra computations.

In these notes we mention new identities for modular forms which are right hand sides of normalized (with respect to Heisenberg vertex operator algebra partition functions) partition and generating functions in the case of self-sewing of the torus to forma a genus two Riemann surface as well as some higher genus cases. For main definition related to vertex operator algebras and computation of their correlation functions see $[3,4,6,16,26,28]$.

\section{GenUs ONE IDENTITIES}

\subsection{Self-sewing of the torus}

Let $M$ be the Heisenberg vertex operator algebra [16], $M \otimes e^{\alpha}$ be its irreducible $M$-module for some $\alpha \in \mathbb{C}$. The torus partition function is given by

$$
Z_{\alpha}^{(1)}(q)=\operatorname{Tr}_{M \otimes e^{\alpha}}\left(q^{L(0)-1 / 24}\right)=\frac{q^{\frac{1}{2} \alpha^{2}}}{\eta(q)},
$$

where $\eta(q)=q^{1 / 24} \prod_{n \geq 1}\left(1-q^{n}\right)$ is the Dedekind eta for modular parameter $q$. Thus

$$
\frac{Z_{\alpha}^{(1)}(q)}{Z_{M}^{(1)}(\tau)}=q^{\frac{1}{2} \alpha^{2}},
$$

where $Z_{M}^{(1)}(\tau)=1 / \eta(q)$ is the genus one Heisenberg rank one partition function. Twisted torus $n$-point function is defined in [37] by

$$
\begin{gathered}
Z_{\alpha}^{(1)}\left(u_{1} \otimes e^{\beta_{1}}, z_{1} ; \ldots ; u_{n} \otimes e^{\beta_{n}}, z_{n} ; q\right) \\
=\operatorname{Tr}_{M \otimes e^{\alpha}}\left(y\left(q_{1}^{L(0)}\left(u_{1} \otimes e^{\beta_{1}}\right), q_{1}\right) \ldots y\left(q_{n}^{L(0)}\left(u_{n} \otimes e^{\beta_{n}}\right), q_{n}\right) q^{L(0)-1 / 24}\right),
\end{gathered}
$$

for formal $q_{i}=e^{z_{i}}$ with $i=1, \ldots, n$. Since $e^{\beta \widehat{q}} M \otimes e^{\alpha}=M \otimes e^{\alpha+\beta}$ it follows that the $n$-point function vanishes when $\sum_{i=1} \beta_{i} \neq 0$.

We next describe a natural generalization of previous results in [21,28]. Firstly, consider the $n$-point functions for $n$ highest weight vectors $\mathbf{1} \otimes e^{\beta_{i}}$, which we abbreviate below to $e^{\beta_{i}}$, for $i=1, \ldots, n$. 
Proposition 1. For $\sum_{i=1}^{n} \beta_{i}=0$ then

$$
\frac{Z_{\alpha}^{(1)}\left(e^{\beta_{1}}, z_{1} ; \ldots ; e^{\beta_{n}}, z_{n} ; q\right)}{Z_{M}^{(1)}(\tau)}=q^{\frac{1}{2} \alpha^{2}} \exp \left(\alpha \sum_{i=1}^{n} \beta_{i} z_{i}\right) \prod_{1 \leq r<s \leq n} K\left(z_{r s}, \tau\right)^{\beta_{r} \beta_{s}},
$$

where $z_{r s}=z_{r}-z_{s}$ and $K(z, \tau)$ is the genus one prime form.

Similarly to [21], we may obtain a closed form for the general $n$-point function (2.1). In particular, we apply standard genus one Zhu recursion theory [34] to reduce (2.1) to an explicit multiple of (2.2) to find

Proposition 2. For $\sum_{i=1}^{n} \beta_{i}=0$ then

$$
\frac{Z_{\alpha}^{(1)}\left(u_{1} \otimes e^{\beta_{1}}, z_{1} ; \ldots ; u_{n} \otimes e^{\beta_{n}}, z_{n} ; q\right)}{Z_{\alpha}^{(1)}\left(e^{\beta_{1}}, z_{1} ; \ldots ; e^{\beta_{n}}, z_{n} ; q\right)}=Q_{\alpha}^{\beta_{1}, \ldots, \beta_{n}}\left(u_{1}, z_{1} ; \ldots u_{n}, z_{n} ; q\right),
$$

where $Q_{\alpha}^{\beta_{1}, \ldots, \beta_{n}}\left(u_{1}, z_{1} ; \ldots u_{n}, z_{n} ; q\right)$ is an explicit sum of elliptic and quasi-modular forms (see [21] for details).

In Propositions 14 and 15 of [28] all torus orbifold $n$-point functions for vectors in $V_{\mathbb{Z}}$ are computed by means of a generating function. In that analysis we made use of an explicit Zhu reduction formula developed for a VOSA with real grading. However, in the present case we do not have an intertwining Zhu reduction formula because of the absence of a commutator formula for interwiners [6]. Instead, here we adopt an alternative approach for computing the intertwining $n$-point functions by exploiting the bosonized formalism. Thus for example, we find

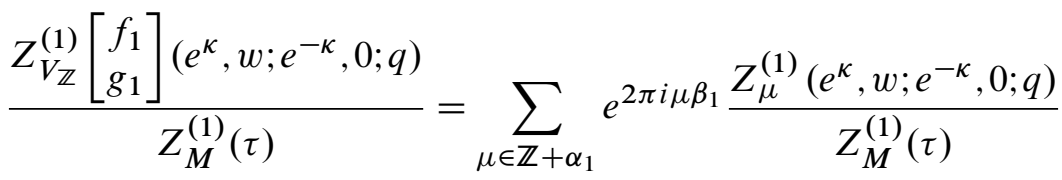

$$
\begin{aligned}
& =\frac{\vartheta\left[\begin{array}{l}
\alpha_{1} \\
\beta_{1}
\end{array}\right](\kappa w, \tau)}{K(w, \tau)^{\kappa^{2}}}
\end{aligned}
$$

for genus one theta series. More generally, we define a generating function for all $n+2$-point functions by the following formal differential form

$$
\begin{gathered}
\mathcal{E}_{n}^{(1)}\left[\begin{array}{l}
f_{1} \\
g_{1}
\end{array}\right]\left(x_{1}, y_{1}, \ldots, x_{n}, y_{n}\right) \\
\equiv Z_{V_{\mathbb{Z}}}^{(1)}\left[\begin{array}{c}
f_{1} \\
g_{1}
\end{array}\right]\left(\psi^{+}, x_{1} ; \psi^{-}, y_{1} ; \ldots ; \psi^{+}, x_{n} ; \psi^{-}, y_{n} ; e^{\kappa}, w ; e^{-\kappa}, 0 ; q\right) \cdot \prod_{i=1}^{n} d x_{i}^{\frac{1}{2}} d y_{i}^{\frac{1}{2}},
\end{gathered}
$$


for $V_{\mathbb{Z}}$ generators $\psi^{ \pm}=e^{ \pm 1}$ alternatively inserted at $x_{i}, y_{i}$ for $i=1, \ldots, n$.

Proposition 3. The generating form (2.1) is given by

$$
\frac{\mathcal{E}_{n}^{(1)}\left[\begin{array}{l}
f_{1} \\
g_{1}
\end{array}\right]\left(x_{1}, y_{1}, \ldots, x_{n}, y_{n}\right)}{Z_{V_{\mathbb{Z}}}^{(1)}\left[\begin{array}{l}
f_{1} \\
g_{1}
\end{array}\right]\left(e^{\kappa}, w ; e^{-\kappa}, 0 ; q\right)}=\operatorname{det} S_{\kappa},
$$

where $S_{\kappa}$ denotes the $n \times n$ matrix with components $S_{\kappa}\left(x_{i}, y_{j}\right)$ for $i, j=1, \ldots, n$ for Szegö kernel.

Finally, we obtain the following generalization of Proposition 15 of [28] concerning the generating properties of (2.1).

Proposition 4. For a pair of square bracket mode twisted Fock vectors

$$
\begin{aligned}
\Psi_{\kappa}\left[\boldsymbol{k}_{1}, \boldsymbol{l}_{2}\right] & =e^{\widehat{\kappa q}} \psi^{+}\left[-k_{11}\right] \ldots \psi^{+}\left[-k_{1 s_{1}}\right] \psi^{-}\left[-l_{21}\right] \ldots \psi^{-}\left[-l_{2 t_{2}}\right] \mathbf{1}, \\
\Psi_{-\kappa}\left[\boldsymbol{k}_{2}, \boldsymbol{l}_{1}\right] & =e^{-\widehat{\widehat{q}}} \psi^{+}\left[-k_{21}\right] \ldots \psi^{+}\left[-k_{2 s_{2}}\right] \psi^{-}\left[-l_{11}\right] \ldots \psi^{-}\left[-l_{1 t_{1}}\right] \mathbf{1},
\end{aligned}
$$

for $p=s_{1}+s_{2}=t_{1}+t_{2}>0$, the generating function $\mathcal{E}_{m}^{(1)}\left[\begin{array}{c}f_{1} \\ g_{1}\end{array}\right]\left(x_{1}, y_{1}, \ldots, x_{m}, y_{m}\right)$ for all torus orbifold intertwining $n+2-$ point functions is given by

$$
\frac{Z_{V_{\mathbb{Z}}}^{(1)}\left[\begin{array}{l}
f_{1} \\
g_{1}
\end{array}\right]\left(\Psi_{\kappa}\left[\boldsymbol{k}_{1}, \boldsymbol{l}_{2}\right], w ; \Psi_{-\kappa}\left[\boldsymbol{k}_{2}, \boldsymbol{l}_{1}\right], 0 ; q\right)}{Z_{V_{\mathbb{Z}}}^{(1)}\left[\begin{array}{l}
f_{1} \\
g_{1}
\end{array}\right]\left(e^{\kappa}, w ; e^{-\kappa}, 0 ; q\right)}=\epsilon \operatorname{det} C_{a b}\left(\boldsymbol{k}_{a}, \boldsymbol{l}_{b}\right),
$$

where

$$
\epsilon=(-1)^{\left(t_{1}+s_{2}\right) t_{2}+\left\lfloor\frac{1}{2} p\right\rfloor} e^{i \pi B \kappa\left(s_{2}-t_{1}\right)},
$$

for some odd integer $B$ and

$$
C_{a b}\left(\boldsymbol{k}_{a}, \boldsymbol{l}_{b}\right)=\left[\begin{array}{ll}
C_{11}\left(\boldsymbol{k}_{1}, \boldsymbol{l}_{1}\right) & C_{12}\left(\boldsymbol{k}_{1}, \boldsymbol{l}_{2}\right) \\
C_{21}\left(\boldsymbol{k}_{2}, \boldsymbol{l}_{1}\right) & C_{22}\left(\boldsymbol{k}_{2}, \boldsymbol{l}_{2}\right)
\end{array}\right],
$$

is a $p \times p$ block matrix with components $C_{a b}\left(k_{a i_{a}}, l_{b j_{b}}\right)$ for $i_{a}=1, \ldots, s_{a}$ and $j_{b}=$ $1, \ldots, t_{b}$ for $a, b=1,2$ for $S_{\kappa}$ expansion coefficients [37].

\section{Genus Two IDENTITIES}

In $[34,43]$ we have proposed a way to derive higher genus generalizations of the classical identities for modular forms. In particular, in [34] we gave a genus two example of the Jacobi product identity. 


\subsection{The self-sewing partition function}

The partition or 0-point function is expressible in terms of the basic genus one twisted 2-point function (2.3) and the infinite Szegó moment matrix $T=T\left(\tau, w, \rho \mid \theta_{1}, \theta_{2}, \phi_{1}, \kappa\right)$ of [37] as follows:

Theorem 1. The genus two continuous orbifold partition function for $V_{\mathbb{Z}}$ on a Riemann surface in the $\rho$-sewing formalism is convergent on $D^{\rho}$ and is given by

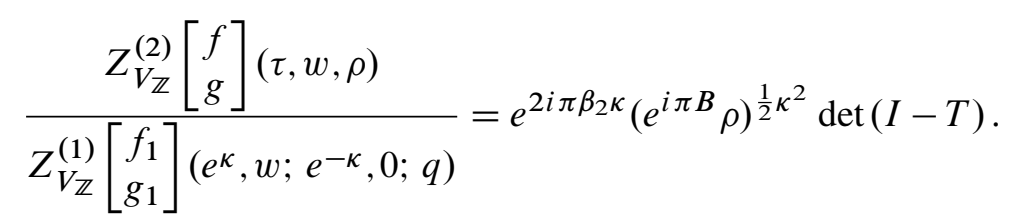

\subsection{An identity for the Szegó kernel}

The "source" genus two Szegó kernel on the torus is defined by [35]

$$
S_{\kappa}(x, y)=\left(\frac{\vartheta_{1}(x-w, \tau) \vartheta_{1}(y, \tau)}{\vartheta_{1}(x, \tau) \vartheta_{1}(y-w, \tau)}\right)^{\kappa} \frac{\vartheta\left[\begin{array}{l}
\alpha_{1} \\
\beta_{1}
\end{array}\right](x-y+\kappa w, \tau)}{\vartheta\left[\begin{array}{l}
\alpha_{1} \\
\beta_{1}
\end{array}\right](\kappa w, \tau) K(x-y, \tau)} d x^{\frac{1}{2}} d y^{\frac{1}{2}},
$$

for $\kappa \neq-\frac{1}{2}$ (with a different expression when $\kappa=-\frac{1}{2}$ given in [35]). Recall also the definition of the Szegố kernel moments [35,37],

$$
\begin{aligned}
G_{a b}(k, l) & =\rho^{\frac{1}{2}\left(k_{a}+l_{b}-1\right)} C_{a b}(k, l), \\
h_{a}(x, k) & =\rho^{\frac{1}{2}\left(k_{a}-\frac{1}{2}\right)} d_{a}(x, k), \\
\bar{h}_{a}(y, k) & =\rho^{\frac{1}{2}\left(k_{a}-\frac{1}{2}\right)} \bar{d}_{a}(y, k),
\end{aligned}
$$

with associated infinite matrix $G=\left(G_{a b}(k, l)\right)$ and row vectors $h(x)=\left(h_{a}(x, k)\right)$ and $\bar{h}(y)=\left(\bar{h}_{a}(y, k)\right)$. Here $d_{a}(y, k)$ are half-order differentials

$$
\begin{aligned}
& d_{a}(x, k)=\frac{1}{2 \pi i} \oint_{\mathcal{C}_{a}\left(y_{a}\right)} y_{a}^{-k_{a}} S_{\kappa}\left(x, y_{a}\right) d y_{a}^{\frac{1}{2}}, \\
& \bar{d}_{a}(y, k)=\frac{1}{2 \pi i} \oint_{\bar{C}_{\bar{a}}\left(x_{\bar{a}}\right)} x_{\bar{a}}^{-k_{a}} S_{\kappa}\left(x_{\bar{a}}, y\right) d x_{\bar{a}}^{\frac{1}{2}},
\end{aligned}
$$

and $C_{a b}(k, l)$ is the infinite block moment matrix

$$
C_{a b}(k, l)=\frac{1}{(2 \pi i)^{2}} \oint_{\complement_{\bar{a}}\left(x_{\bar{a}}\right)} \oint_{\complement_{b}\left(y_{b}\right)}\left(x_{\bar{a}}\right)^{-k_{a}}\left(y_{b}\right)^{-l_{b}} S_{\kappa}\left(x_{\bar{a}}, y_{b}\right) d x_{\bar{a}}^{\frac{1}{2}} d y_{b}^{\frac{1}{2}} .
$$

Thus the genus two Szegô kernel on the self-sewn torus is given by

$$
S^{(2)}(x, y)=S_{\kappa}(x, y)+\xi h(x) D^{\theta}(I-T)^{-1} \bar{h}^{T}(y),
$$


where $\bar{h}^{T}(y)$ denotes the transpose of the infinite row vector $\bar{h}(y)$.

$$
\begin{gathered}
T=\xi G D^{\theta_{2}}, \\
D^{\theta_{2}}(k, l)=\left[\begin{array}{cc}
\theta_{2}^{-1} & 0 \\
0 & -\theta_{2}
\end{array}\right] \delta(k, l) .
\end{gathered}
$$

Using (3.9) we define matrices

$$
\begin{aligned}
& S^{(2)}=\left(S^{(2)}\left(x_{i}, y_{j}\right)\right), \quad S_{\kappa}=\left(S_{\kappa}\left(x_{i}, y_{j}\right)\right), \\
& H=\left(\left(h\left(x_{i}\right)\right)(k, a)\right), \quad \bar{H}^{T}=\left(\left(\bar{h}\left(y_{i}\right)\right)(l, b)\right)^{T} .
\end{aligned}
$$

$S^{(2)}$ and $S_{\kappa}$ are finite matrices indexed by $i, j=1, \ldots, n ; H$ is semi-infinite with $n$ rows indexed by $i$ and columns indexed by $k \geq 1$ and $a=1,2$ and $\bar{H}^{T}$ is semiinfinite with rows indexed by $l \geq 1$ and $b=1,2$ and with $n$ columns indexed by $j$. Similarly to Proposition 3 in [35] we have

\section{Proposition 5.}

$$
\operatorname{det}\left[\begin{array}{cc}
S_{\kappa} & -\xi H D^{\theta_{2}} \\
\bar{H}^{T} & I-T
\end{array}\right]=\operatorname{det} S^{(2)} \operatorname{det}(I-T),
$$

with $T, D^{\theta_{2}}$ of (3.10) and (3.11).

\subsection{The genus two n-point generating form}

Similarly to the genus one situation (2.1), we define a genus two continuous orbifold generating differential form by

$$
\begin{gathered}
\mathcal{E}_{n}^{(2)}\left[\begin{array}{c}
f \\
g
\end{array}\right]\left(x_{1}, y_{1}, \ldots, x_{n}, y_{n}\right) \\
=Z_{V_{\mathbb{Z}}}^{(2)}\left[\begin{array}{c}
f \\
g
\end{array}\right]\left(\psi^{+}, x_{1} ; \psi^{-}, y_{1} ; \ldots, \psi^{+}, x_{n} ; \psi^{-}, y_{n} ; \tau, w, \rho\right) \prod_{i=1}^{n} d x_{i}^{\frac{1}{2}} d y_{i}^{\frac{1}{2}} .
\end{gathered}
$$

Then we have

Theorem 2. The generating form (3.3) is given by

$$
\frac{\mathscr{E}_{n}^{(2)}\left[\begin{array}{l}
f \\
g
\end{array}\right]\left(x_{1}, y_{1}, \ldots, x_{n}, y_{n}\right)}{Z_{V_{\mathbb{Z}}}^{(2)}\left[\begin{array}{l}
f \\
g
\end{array}\right](\tau, w, \rho)}=\operatorname{det} S^{(2)},
$$

for $n \times n$ matrix $S^{(2)}$ of (3.12) and $Z_{V_{\mathbb{Z}}}^{(2)}\left[\begin{array}{l}f \\ g\end{array}\right](\tau, w, \rho)$ is the genus two twisted partition function (3.1). 
Normalized relative to the genus two partition function, the 2-point function for $\psi^{+}$and $\psi^{-}$is thus given by the Szegó kernel and more generally, the generating function is a Szegő kernel determinant. This agrees with the assumed form in [30] or as found by string theory methods using a Schottky parametrization in [5] and in the genus two $\epsilon$-sewing scheme [35].

\subsection{Genus two Heisenberg partition function}

We may compute the genus two twisted partition and generating functions in an alternative way by use of a bosonic basis $u \otimes e^{\nu} \in V_{\mathbb{Z}+\kappa}$ for $u \in M$ and $v \in \mathbb{Z}+\kappa$. In particular, we can immediately exploit and extend the results for lattice VOAs in [24].

Let us firstly recall from $[22,24]$ the following definitions:

$$
\begin{aligned}
P_{2}(\tau, z) & =\wp(\tau, z)+E_{2}(\tau) \\
& =\frac{1}{z^{2}}+\sum_{k=2}^{\infty}(k-1) E_{k}(\tau) z^{k-2},
\end{aligned}
$$

for Weierstrass function $\wp(\tau, z)$ and Eisenstein series $E_{k}(\tau)$. We define

$$
P_{k+1}(\tau, z)=-\frac{1}{k} \partial_{z} P_{k}(\tau, z),
$$

for $k \geq 2$ and for $k, l \geq 1$

$$
\begin{aligned}
C(k, l, \tau) & =(-1)^{k+1} \frac{(k+l-1) !}{(k-1) !(l-1) !} E_{k+l}(\tau), \\
D(k, l, \tau, z) & =(-1)^{k+1} \frac{(k+l-1) !}{(k-1) !(l-1) !} P_{k+l}(\tau, z), \\
R_{a b}(k, l) & =-\frac{\rho^{(k+l) / 2}}{\sqrt{k l}}\left[\begin{array}{cc}
D(k, l, \tau, w) & C(k, l, \tau) \\
C(k, l, \tau) & D(l, k, \tau, w)
\end{array}\right] .
\end{aligned}
$$

Theorems 5.1 and 5.6 of [24] tell us that:

Theorem 3. The genus two normalized partition function for the Heisenberg VOA $M$ in the self-sewing scheme is

$$
\frac{Z_{M}^{(2)}(\tau, w, \rho)}{Z_{M}^{(1)}(\tau)}=(\operatorname{det}(1-R))^{-1 / 2},
$$

where $Z_{M}^{(2)}(\tau, w, \rho)$ is holomorphic on $D^{\rho}$. 


\subsection{Lattice VOA}

Theorem 6.1 of [24] concerns the genus two partition function for a lattice VOA. We obtain a natural generalization for a twisted genus two partition function defined by

$$
Z_{\mu, \nu}^{(2)}(\tau, w, \rho)=\sum_{\Psi_{\nu} \in M \otimes e^{\nu}} Z_{\mu}^{(1)}\left(\Psi_{\nu}, w ; \bar{\Psi}_{\nu}, 0 ; q\right)
$$

for $\mu, v \in \mathbb{C}$ where the sum is taken over a $M \otimes e^{v}$ basis, $\bar{\Psi}_{v}$ is the square bracket dual (with respect to a non-generate form, see $[34,37]$ ) and the summand is a genus one 2-point function (2.1) for $\mathcal{M}$. We find that the normalized partition function is given by the theta-function.

\section{Theorem 4.}

$$
\frac{Z_{\mu, v}^{(2)}(\tau, w, \rho)}{Z_{M}^{(2)}(\tau, w, \rho)}=e^{i \pi\left(\mu^{2} \Omega_{11}^{(2)}+2 \mu \nu \Omega_{12}^{(2)}+v^{2} \Omega_{22}^{(2)}\right)},
$$

where $\Omega^{(2)}$ is the genus two period matrix.

We are now able to compute the genus two twisted partition function (3.1) by use of a bosonic basis to obtain

Theorem 5. The genus two normalized twisted partition function for $V_{\mathbb{Z}}$ on a Riemann surface in the self-sewing formalism is given by

$$
\frac{Z_{V_{\mathbb{Z}}}^{(2)}\left[\begin{array}{l}
f \\
g
\end{array}\right](\tau, w, \rho)}{Z_{M}^{(2)}(\tau, w, \rho)}=\vartheta\left[\begin{array}{l}
\alpha \\
\beta
\end{array}\right]\left(\Omega^{(2)}\right),
$$

for genus two Riemann theta function with some characteristics $\alpha_{1}, \alpha_{2}=\kappa, \beta_{1}, \beta_{2}$ and where $Z_{M}^{(2)}(\tau, w, \rho)$ is the genus two Heisenberg partition function.

\subsection{Generalizations of classical identities for modular forms}

In [34] we proved a generalization of the classical Jacobi identity suitable for genus two. By comparison of the direct computation via algebraic technique [21]-[26], [34]-[38] of the partition and $n$-point functions with the bosonization technique [21], we obtain $[34,37]$ the genus two version of the Jacobi product and Frobenius-Fay trisecant identities. Comparing with the fermionic expression (3.1) and noting that

$$
\left(e^{i \pi B} \rho\right)^{\frac{1}{2} \kappa^{2}} \frac{Z_{V_{\mathbb{Z}}}^{(1)}\left[\begin{array}{c}
f_{1} \\
g_{1}
\end{array}\right]\left(e^{\kappa}, w ; e^{-\kappa}, 0 ; q\right)}{Z_{M}^{(1)}(\tau)}=\left(\frac{e^{i \pi B} \rho}{K(w, \tau)^{2}}\right)^{\frac{1}{2} \kappa^{2}} \vartheta^{(1)}\left[\begin{array}{l}
\alpha_{1} \\
\beta_{1}
\end{array}\right](\kappa w, \tau),
$$

we obtain a genus two analogue of the classical Jacobi triple product identity for the elliptic theta function (separate to a similar identity shown in [34]) as follows: 
Theorem 6. The ratio of genus two and genus one Riemann theta functions on $D^{\rho}$ is given by

$$
\frac{\vartheta^{(2)}\left[\begin{array}{l}
\alpha \\
\beta
\end{array}\right]\left(\Omega^{(2)}\right)}{\vartheta^{(1)}\left[\begin{array}{l}
\alpha_{1} \\
\beta_{1}
\end{array}\right](\kappa w, \tau)}=e^{2 i \pi \beta_{2} \kappa}\left(\frac{e^{i \pi B} \rho}{K(w, \tau)^{2}}\right)^{\frac{1}{2} \kappa^{2}} \operatorname{det}(I-T) \operatorname{det}(I-R)^{\frac{1}{2}} .
$$

As suggested in [30], we also remark that Fay's Trisecant Identity at genus two follows by comparing the generating function $\mathscr{E}_{n}^{(2)}\left[\begin{array}{l}f \\ g\end{array}\right]\left(x_{1}, y_{1}, \ldots, x_{n}, y_{n}\right)$ of (3.3) to its form in terms of a bosonic basis.

Thus we see that even at the partition function level, automorphic objects (in particular, ratios of $\theta$-functions) can be related to certain functions expressed via determinants [23], [28], [34], [36] containing data (coefficients in expansions of regular parts of ordinary or half-order (e.g., Szegő kernel) differentials) coming from sewing of lower genus Riemann surfaces. These identities reflect automorphic-differential outcome of the boson-fermion correspondence.

\section{HighER GENUS IDENTITIES}

\subsection{The partition functions for Heisenberg VOA}

Let $Z_{M}^{(2)}\left(\Omega^{(2)}\right)$ be the genus two partition function for the rank one free Heisenberg VOA $M$ [23]. It has been computed in [23,25]

$$
\frac{Z_{M}^{(2)}\left(\Omega^{(2)}\right)}{Z_{M}^{(1)}\left(\tau_{1}\right) Z_{M}^{(1)}\left(\tau_{2}\right)}=\operatorname{det}\left(I-A_{1}^{(1)} A_{2}^{(1)}\right)^{-1 / 2} \text {. }
$$

Here $A_{a}^{(1)}$ for $a=1,2$ are infinite matrices with components indexed by $k, l \geq 1$ $[23,24]$,

$$
A_{a}\left(k, l, \tau_{a}, \epsilon\right)=\epsilon^{(k+l) / 2} \frac{(-1)^{k+1}(k+l-1) !}{\sqrt{k l}(k-1) !(l-1) !} E_{k+l}\left(\tau_{a}\right),
$$

where $\epsilon$ is Riemann surface sewing scheme parameter [24,40], and $E_{n}(\tau)$ are the Eisenstein series [31]. Note that $E_{n}(\tau)$ appear explicitly as a result of vertex operator algebra computation.

At genus $g$, in two curves sewing formalism, we obtain [38] similar formula for the rank two Heisenberg VOA module

$$
\frac{Z_{M_{2}}^{(g)}}{Z_{M_{2}}^{\left(g_{1}\right)} Z_{M_{2}}^{\left(g_{2}\right)}}=\operatorname{det}\left(I-A^{\left(g_{1}, g_{2}\right)}\right)^{-1},
$$


where $Z_{M_{2}}^{(g)}$ is a non-vanishing holomorphic function on the sewing domain, and is automorphic with respect to $S L(2, \mathbb{Z}) \times \ldots \times S L(2, \mathbb{Z}) \subset S p(2 g, \mathbb{Z})$ with automorphy factor $\operatorname{det}\left(C \Omega^{(g)}+D\right)^{-1}$, and a multiplier system. Here $A^{\left(g_{1}, g_{2}\right)}$ is an infinite moment matrix containing genus $g$ sewing data, and $\Omega^{(g)}$ is the genus $g$ period matrix. Automorphic properties of the above mentioned genus two partition functions follow $[34,37]$ from the structure of the determinant and lower genus partition functions in (4.3). An alternative construction of the genus $g$ partition function for the Heisenberg vertex operator algebra is performed in [33] via the Schottky parameterization.

\subsection{The free fermion partition functions}

The genus one free fermion twisted partition function is given by [16,28]

$$
Z_{V_{Z}}^{(1)}\left[\begin{array}{l}
f \\
g
\end{array}\right](\tau)=q^{\alpha^{2} / 2-1 / 24} \prod_{l \geq 1}\left(1-\theta^{-1} q^{l-\frac{1}{2}+\alpha}\right)\left(1-\theta q^{l-\frac{1}{2}-\alpha}\right),
$$

which represents a form of the genus one Jacobi triple identity [16]. It is clearly a modular invariant object. This partition function can be also obtained [35,37] in the form analogous to (4.3) as a determinant of an infinite matrix containing moments of corresponding genus zero differentials when considering a self-sewing of the sphere.

Genus $g$ formula for the partition function can be also conjectured [38]:

$$
\frac{Z_{V_{\mathbb{Z}}}^{(g)}\left[\begin{array}{l}
f^{(g)} \\
g^{(g)}
\end{array}\right]\left(\Omega^{(g)}\right)}{Z_{V_{\mathbb{Z}}}^{\left(g_{1}\right)}\left[\begin{array}{c}
f^{\left(g_{1}\right)} \\
g^{\left(g_{1}\right)}
\end{array}\right]\left(\Omega^{\left(g_{1}\right)}\right) Z_{V_{\mathbb{Z}}}^{\left(g_{2}\right)}\left[{ }_{g^{\left(g_{2}\right)}}^{f^{\left(g_{2}\right)}}\right]\left(\Omega^{\left(g_{2}\right)}\right)}=\operatorname{det}\left(I-Q^{\left(g_{1}, g_{2}\right)}\right),
$$

where $g=g_{1}+g_{2}, Q^{\left(g_{1}, g_{2}\right)}$ is an infinite matrix, and $\Omega^{(g)}, \Omega^{\left(g_{1}\right)}, \Omega^{\left(g_{2}\right)}$ are period matrices for a sewn genus $g$ curve and lower genus curves of genuses $g_{1}, g_{2}$. In particular in [34] we proved that the genus two twisted partition function for the rank two fermion VOSA is given by

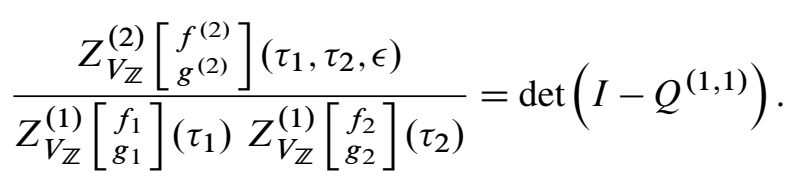

\section{DifFERENTIAL SIDE: WARD IDENTITIES}

Another group of identities are used to prove an autmorphic-differential correspondence [45].

Let $X$ be a compact connected simply connected algebraic curve. As it is explained in $[1,3,13]$, twisted $D$-modules on the moduli stack $B u n_{G}$ of $G$-bundles over $X$ arise in conformal field theories as sheaves of conformal blocks on the moduli space $\mathcal{M}_{g, n}$ of pointed complex curves of genus $g$. These $\mathscr{D}$-modules encode chiral correlation functions of a model. For CFT's with Lie algebraic symmetries 
[13], correlation functions satisfy Ward identities which involve $[2,11]$ projectively flat connections on a bundle of conformal blocks. For sheaves of conformal blocks on $B u_{G}$, one obtains a projective connection in the bundle or the structure of a twisted $\mathscr{D}$-module on the sheaf of conformal blocks.

Based on algebraic properties of VOA modules, we show [36] that certain normalized (i.e., divided by appropriate Heisenberg VOA partition function, e.g., (5.1), (5.2) and (5.5), see subsections 5.1 and 5.2) generating differentials for $n$-point functions can be represented as differential operators acting on functions with nice automorphic properties (such as $\theta$-functions). Then such differential operators turn out to be connections in bundles over Riemann surfaces. This construction can be extended to differentials associated to arbitrary $n$-point functions (e.g., (5.3), see subsection 5.2) even on higher genus Riemann surfaces. This construction represents another side of the geometric correspondence for vertex algebras.

\subsection{The partition functions for free fermionic VOSA}

Let $V_{\mathbb{Z}}$ be a free fermionic vertex operator superalgebra $V$ module [28]. The normalized form of the genus one twisted partition function (see (4.4) in subsection 4.2) for the rank two free fermionic vertex operator superalgebra (see, e.g., [16], [28]) can be also expressed as

$$
\frac{Z_{V_{\mathbb{Z}}[1}^{(1)}\left[\begin{array}{l}
f \\
g
\end{array}\right](\tau)}{Z_{M}^{(1)}(\tau)}=e^{-2 \pi i \alpha \beta} \cdot \vartheta^{(1)}\left[\begin{array}{l}
\alpha \\
\beta
\end{array}\right](\tau),
$$

for the torus theta function with characteristics $\alpha, \beta$. The genus two normalized twisted partition function (4.6) can be computed in the bosonization formalism to obtain $[21,34]$

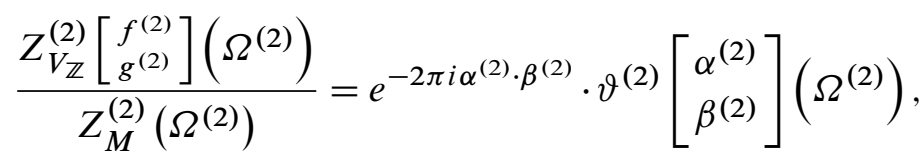

for the genus two Riemann theta function with characteristics $\alpha^{(2)}=\left(\alpha_{1}, \alpha_{2}\right), \beta^{(2)}=$ $\left(\beta_{1}, \beta_{2}\right)$ where $Z_{M}^{(2)}\left(\Omega^{(2)}\right)$ is the genus two Heisenberg partition function. Natural formulas relating the free fermion normalized twisted partition functions and corresponding theta-functions are also available at higher genus [38].

\subsection{Free fermion genus two twisted generating $n$-point functions}

For $v_{i} \in V$, and points $z_{i}, i=1, \ldots n$, on a genus $g$ curve, let us introduce [34] the following differentials:

$$
\mathcal{F}_{V_{\mathbb{Z}, n}}^{(g)}\left[\begin{array}{l}
f^{(g)} \\
g^{(g)}
\end{array}\right]\left(\left(v_{1}, z_{1}\right), \ldots,\left(v_{n}, z_{n}\right)\right)
$$




$$
\equiv F_{V_{\mathbb{Z}}, n}^{(g)}\left[\begin{array}{l}
f^{(g)} \\
g^{(g)}
\end{array}\right]\left(\left(v_{1}, z_{1}\right), \ldots,\left(v_{n}, z_{n}\right)\right) \prod_{i=1}^{n} d z_{i}^{w t\left(v_{i}\right)},
$$

where $w t\left(v_{i}\right)$ are weights of the states $v_{i}$, [16]. In [34] we proved that for $g=2$ the generating differential for all rank two free fermion VOSA $n$-point functions is given by the differential

$$
\begin{gathered}
\mathcal{E}_{V_{\mathbb{Z}}, n}^{(2)}\left[\begin{array}{l}
f^{(2)} \\
g^{(2)}
\end{array}\right]\left(x_{1}, y_{1}, \ldots, x_{n}, y_{n}\right) \\
\equiv F_{V_{\mathbb{Z}}, n}^{(2)}\left[\begin{array}{c}
f^{(2)} \\
g^{(2)}
\end{array}\right]\left(\left(\psi^{+}, x_{1}\right),\left(\psi^{-}, y_{1}\right) \ldots,\left(\psi^{+}, x_{n}\right),\left(\psi^{-}, y_{n}\right)\right) \cdot \prod_{i, j=1}^{n} d x_{i}^{1 / 2} d y_{j}^{-1 / 2},
\end{gathered}
$$

where $F_{V_{\mathbb{Z}, n}}^{(2)}\left[\begin{array}{l}f^{(2)} \\ g^{(2)}\end{array}\right]\left(\left(\psi^{+}, x_{1}\right),\left(\psi^{-}, y_{1}\right) \ldots,\left(\psi^{+}, x_{n}\right),\left(\psi^{-}, y_{n}\right)\right)$ is the genus two $2 n$ point function in coordinates $x_{i}, y_{i}, i=1, \ldots, n ; \psi^{ \pm}$are generating states, and $f^{(2)}$, $g^{(2)}$ being vectors containing pairs of $V$-twisting automorphisms. Then its normalized form is given by

$$
\frac{\mathcal{E}_{V_{\mathbb{Z}, n}}^{(2)}\left[\begin{array}{c}
f \\
g
\end{array}\right]\left(x_{1}, y_{1}, \ldots, x_{n}, y_{n}\right)}{Z_{M}^{(2)}}=e^{-2 \pi i \alpha \cdot \beta} \operatorname{det} S^{(2)} \cdot \vartheta^{(2)}\left[\begin{array}{c}
\alpha \\
\beta
\end{array}\right]\left(\Omega^{(2)}\right),
$$

where elements of the matrix $S^{(2)}=\left[S^{(2)}\left[\begin{array}{l}\theta \\ \phi\end{array}\right]\left(x_{i}, y_{j}\right)\right], i, j=1, \ldots, n$ are genus two Szegő kernels, i.e.,

$$
S^{(2)}\left[\begin{array}{l}
\theta^{(2)} \\
\phi^{(2)}
\end{array}\right]\left(x_{i}, y_{j}\right)=K^{(2)}\left[\begin{array}{l}
\theta^{(2)} \\
\phi^{(2)}
\end{array}\right]\left(x_{i}, y_{j}\right) d x_{i}^{1 / 2} d y_{j}^{1 / 2},
$$

and $K^{(2)}\left[\begin{array}{l}\theta \\ \phi\end{array}\right]\left(z_{1}, z_{2}\right)$ is the functional part of the genus two prime form [9].

\subsection{Genus two one-point Virasoro vector function}

In [34] (see also [37]) we gave an example of a genus two generating function relation containing a flat connection over a Riemann surface. In [34] we computed the one-point function for the Virasoro vector $\widetilde{\omega}=\frac{1}{2} a[-1] a$, where $a$ is the Heisenberg element in the bosonized version of the rank two free fermion VOSA on a genus two Riemann surface obtained in the sewing procedure of two tori.

The Virasoro vector can be written as $\widetilde{\omega}=\frac{1}{2}\left(\psi^{+}[-2] \psi^{-}+\psi^{-}[-2] \psi^{+}\right)$for the rank two free fermion generators $\psi^{ \pm}$in the Zhu equivalent VOSA formulation. Let $w, z$ be two distinct points on a genus two Riemann surface. To compute a one point 
function we use the normalized generating form

$$
\frac{\mathscr{E}_{V_{\mathbb{Z}}, 1}^{(2)}\left[\begin{array}{l}
f^{(2)} \\
g^{(2)}
\end{array}\right](w, z)}{Z_{V_{\mathbb{Z}}}^{(2)}\left[\begin{array}{l}
f^{(2)} \\
g^{(2)}
\end{array}\right]}=S^{(2)}\left[\begin{array}{l}
\theta^{(2)} \\
\phi^{(2)}
\end{array}\right](w, z)
$$

Then we find [36]

$$
\begin{aligned}
& \frac{\partial_{w} F_{V_{\mathbb{Z}}, 2}^{(2)}\left[\begin{array}{c}
f^{(2)} \\
g^{(2)}
\end{array}\right]\left(\psi^{+}, w ; \psi^{-}, z\right)}{Z_{V_{\mathbb{Z}}}^{(2)}\left[\begin{array}{l}
f^{(2)} \\
g^{(2)}
\end{array}\right]}=\frac{\partial_{w} F_{V_{\mathbb{Z}}, 1}^{(2)}\left[\begin{array}{c}
f^{(2)} \\
g^{(2)}
\end{array}\right]\left(Y\left[\psi^{+}, w-z\right] \psi^{-}, z\right)}{Z_{V_{\mathbb{Z}}}^{(2)}\left[\begin{array}{l}
f^{(2)} \\
g^{(2)}
\end{array}\right]}
\end{aligned}
$$

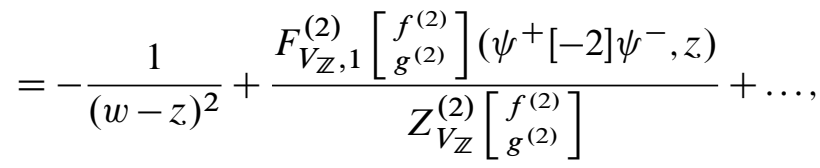

and similarly for $\partial_{z}$. Due to (5.6) it follows that the Virasoro vector one-point differential form is

$$
\frac{\widetilde{F}_{V_{\mathbb{Z}}, 1}^{(2)}\left[\begin{array}{l}
f^{(2)} \\
g^{(2)}
\end{array}\right](\widetilde{\omega}, z)}{Z_{V_{\mathbb{Z}}}^{(2)}\left[\begin{array}{l}
f^{(2)} \\
g^{(2)}
\end{array}\right]}=d z^{2} \lim _{w \rightarrow z}\left(\frac{1}{(w-z)^{2}}+\frac{1}{2}\left(\partial_{w}-\partial_{z}\right) K^{(2)}(w, z)\right) .
$$

An alternative expression [34] for this is given below in (5.8).

\subsection{Flat connections}

Introduce the differential operator [39], [21], [34]

$$
\mathscr{D}^{(g)}=\frac{1}{2 \pi i} \sum_{1 \leq i \leq j \leq g} v_{i}^{(g)}(x) v_{j}^{(g)}(x) \frac{\partial}{\partial \Omega_{i j}^{(g)}} .
$$

It includes holomorphic 1-forms $v_{i}^{(g)}$ as well as derivative with respect to the genus $g$ Riemann surface period matrix $\Omega^{(g)}$. The genus $g$ projective connection $s^{(g)}$ is defined by [15]

$$
s^{(g)}(x)=6 \lim _{x \rightarrow y}\left(\omega^{(g)}(x, y)-\frac{d x d y}{(x-y)^{2}}\right),
$$

where $\omega^{(g)}$ is the meromorphic differential of the second kind on a Riemann surface. The projective connection $s^{(g)}(x)$ is not a global 2-form but rather transforms under a general conformal transformation $x \rightarrow \phi(x)$ as follows $s^{(g)}(\phi(x))=s^{(g)}(x)-$ $\{\phi ; x\} d x^{2}$, where $\{\phi ; x\}=\frac{\phi^{\prime \prime \prime}}{\phi^{\prime}}-\frac{3}{2}\left(\frac{\phi^{\prime \prime}}{\phi^{\prime}}\right)^{2}$ is the Schwarzian derivative. 


\subsection{Genus two Ward identity}

It turns out that the Virasoro one-point differential form on genus two Riemann surface can be represented by means of the Ward identity as a flat connection in certain bundle. Let $Z_{M}^{(2)}$ be the genus two partition function for the rank one Heisenberg VOA $M$ (see explicit formula (4.1)). Using results of [25] we proved in [34] that the Virasoro one-point normalized differential form for the rank two fermion VOSA satisfies the genus two Ward identity

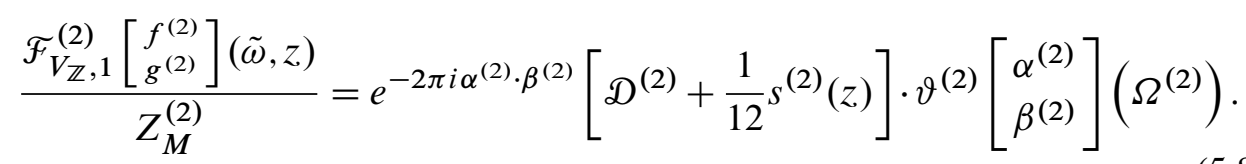

Here the expression for the normalized genus two one-point differential form is represented as the action of a differential operator on an automorphic function. One can generalize [38] (5.8) to genus $g$ formula. Note that the Ward identities coherent with the above formula appeared previously in $[8,17]$. In contrast to the pure algebraicgeometry $[11,17,32,39]$ and physics approaches $[2,11]$, the Ward identity shows up from algebraic properties of corresponding vertex algebra. The Ward identities were also used in [12] to establish a version of the geometric Langlands correspondence for Kac-Moody Lie algebras at critical level.

One can also represent the genus two free fermion generating differentials (5.4) (see subsection 5.2) using projective connections [34]. In particular, due to definitions of the genus $g$ Szegó kernel and prime form [9], it is possible to rewrite more general formula for the normalized twisted generating differential (5.5) for all $n$-point free fermion functions as a quite complicated operator acting on a theta function in a form of (5.8). Similar Ward identities are also available at higher genus cases [14, 38]. Another interesting way to represent the formula (5.5) (and thus (5.8), (5.7), see subsection 5.3) is by using an alternative formulation of the prime form given in [10]. This point will be discussed in [45].

Computations involving boson-fermion correspondence compared to direct computations of generating functions give [28] us various higher genus generalizations of the Frobenius-Fay identities [9]. Using the Ward identity form (e.g., (5.8)) for generating differentials, one is also able to deduce projective connection form for those identities by comparison with pure vertex operator algebra computations.

In examples given above we see that for fermionic VOSAs there is some sense to write expressions for the partition and generating functions in normalized form sinse then differential (projective connection structure) is clear. On the other hand, unnormalized form reflects the automorphic side of the correspondence.

Another interesting direction in generating identities for modular form is related to Jacobi forms via vertex operator algebras [18-20]. We find in this way analogues of the Frobenius-Fay identities [44] for Jacobi forms. 


\section{ACKNOWLEDGEMENT}

We would like to express our gratitude to the organizers of the Conference Representation Theory XIII, Dubrovnik, Croatia, 2013. This text is based on the author's talk on the Conference.

\section{REFERENCES}

[1] A. Beilinson and V. Drinfeld, Chiral algebras, ser. Cooloquim Publications. Providence, RI: American Mathematical Society, 2004.

[2] A. Belavin, A. Polyakov, and A. Zamolodchikov, "Infinite conformal symmetries in twodimensional quantum field theory," Nucl. Phys. B, vol. 241, pp. 333-380, 1984.

[3] D. Ben-Zvi and E. Frenkel, Vertex algebras and algebraic curves, 2nd ed., ser. Mathematical Surveys and Monographs. Providence, RI: American Mathematical Society, 2004.

[4] R. Borcherds, "Vertex algebras, Kac-Moody algebras and the Monster," Proc. Nat. Acad. Sc., vol. 83, pp. 3068-3071, 1986.

[5] F. Di Vecchia, P.and Pezzella, M. Frau, K. Hornfeck, A. Lerda, and S. Sciuto, " $n$-point $g$-loop vertex for a free fermionic theory with arbitrary spin," Nucl. Phys. B, vol. 333, pp. 635-700, 1990.

[6] C. Dong and J. Lepowsky, Generalized Vertex Algebras and Relative Vertex Operators, ser. Progress in Mathematics. Boston, MA: Birkhäuser, 1993, vol. 112.

[7] C. Dong, H. Li, and M. G., "Modular-invariance of trace functions in orbifold theory and generalized moonshine," Comm. Math. Phys., vol. 214, pp. 1-56, 2000.

[8] T. Eguchi and H. Ooguri, "Conformal and current algebras on a general Riemann surface," Nucl. Phys. B, vol. 282, pp. 308-328, 1987.

[9] J. Fay, Theta Functions on Riemann Surfaces, ser. Lecture Notes in Mathematics. Berlin-New York: Springer-Verlag, 1973, vol. 352.

[10] J. Fay, Kernel functions, analytic torsion, and moduli spaces, ser. Mem. Amer. Math. Soc. Providence, RI: Amer. Math. Soc., 1992, vol. 96.

[11] D. Freidan and S. Shenker, "The analytic geometry of two dimensional conformal field theory," Nucl. Phys. B, vol. 281, pp. 509-545, 1987.

[12] E. Frenkel, Langlands Correspondence for Loop Groups, ser. Cambridge Studies in Advanced Mathematics. Cambridge University Press, 2007, vol. 103.

[13] E. Frenkel, Lectures on the Langlands Program and Conformal Field Theory, ser. Frontiers in number theory, physics, and geometry. Berlin: Springer, 2007, vol. II.

[14] T. Gilroy and M. Tuite, "To appear," 2014.

[15] R. Gunning, Lectures on Riemann Surfaces. Princeton: Princeton Univ. Press, 1966, vol. 134.

[16] V. Kac, Vertex Operator Algebras for Beginners, ser. University Lecture Series. Providence: AMS, 1998, vol. 10.

[17] N. Kawamoto, Y. Namikawa, A. Tsuchiya, and Y. Yamada, "Geometric realization of conformal field theory on Riemann surfaces," Commun. Math. Phys., vol. 116, pp. 247-308, 1988.

[18] M. Krauel, "A Jacobi theta series and its transformation laws," Int. J. Number Theory, vol. 10, no. 6, pp. 1343-1354, 2014.

[19] M. Krauel and M. G., "Vertex operator algebras and weak Jacobi forms," Internat. J. Math., vol. 23, no. 6, p. 1250024, 2012.

[20] M. Krauel and M. G., "Jacobi trace functions in the theory of vertex operator algebras," arXiv:1309.5720, 2013.

[21] G. Mason and M. Tuite, "Torus chiral n-point functions for free boson and lattice vertex operator algebras," Comm. Math. Phys., vol. 235, no. 1, pp. 47-68, 2003. 
[22] G. Mason and M. Tuite, Chiral algebras and partition functions. Lie algebras vertex operator algebras and their applications, ser. Contemp. Math. Providence, RI: Amer. Math. Soc., 2007, vol. 442.

[23] G. Mason and M. Tuite, "The genus two partition function for free bosonic and lattice vertex operator algebras," arXiv:0712.0628, 2007.

[24] G. Mason and M. Tuite, "On genus two Riemann surfaces formed from sewn tori," Comm. Math. Phys., vol. 270, no. 3, pp. 587-634, 2007.

[25] G. Mason and M. Tuite, "Free bosonic vertex operator algebras on genus two Riemann surfaces I," Comm. Math. Phys., vol. 300, pp. 673-713, 2010.

[26] G. Mason and M. Tuite, Vertex operators and modular forms. A window into zeta and modular physics, ser. Math. Sci. Res. Inst. Publ. Cambridge: Cambridge Univ. Press, 2010, vol. 57.

[27] G. Mason and M. Tuite, "Free bosonic vertex operator algebras on genus two Riemann surface II," in Conformal Field Theory, Conformal Field Theory,Automorphic Forms and Related Topics, ser. Contributions in Mathematical and Computational Sciences, vol. 8. Berlin Heidelberg: SpringerVerlag, 2014.

[28] G. Mason, M. Tuite, and A. Zuevsky, "Torus $n$-point functions for $\mathbb{R}$-graded vertex operator superalgebras and continuous fermion orbifolds," Comm. Math. Phys., vol. 283, no. 2, pp. 305-342, 2008.

[29] M. Miyamoto, "A modular invariance on the theta functions defined on vertex operator algebras," Duke Math. J., vol. 101, no. (2), pp. 221-236, 2000.

[30] A. Raina, "Fay's trisecant identity and conformal field theory," Comm. Math. Phys., vol. 122, pp. 625-641, 1989.

[31] J.-P. Serre, A course in arithmetic, ser. Math. Sci. Res. Inst. Publ. Berlin: Springer-Verlag, 1978, vol. 57.

[32] A. Tsuchiya and Y. Ueno, K.and Yamada, Conformal field theory on universal family of stable curves with gauge symmetries, ser. Advanced Studies in Pure Math. Tokyo: Kinokuniya Company Ltd., 1989, vol. 19.

[33] M. P. Tuite and A. Zuevsky, "The bosonic vertex operator algebra on a genus g Riemann surface," RIMS Kokyuroko, vol. 1756, pp. 81-93, 2011.

[34] M. P. Tuite and A. Zuevsky, "Genus two partition and correlation functions for fermionic vertex operator superalgebras I,” Comm. Math. Phys., vol. 306, no. 2, pp. 419-447, 2011.

[35] M. P. Tuite and A. Zuevsky, "The Szegő kernel on a sewn Riemann surface," Comm. Math. Phys., vol. 306, no. 3, pp. 617-645, 2011.

[36] M. P. Tuite and A. Zuevsky, "A generalized vertex operator algebra for Heisenberg intertwiners," J. Pure and Applied Alg., vol. 216, no. 6, pp. 1253-1492, 2012.

[37] M. P. Tuite and A. Zuevsky, "Genus two partition and correlation functions for fermionic vertex operator superalgebras II," to appear in J. Math. Phys., arxiv.org/abs/1308.2441, 2013.

[38] M. P. Tuite and A. Zuevsky, "The Heisenberg vertex operator algebra on a genus $g$ Riemann surface," RIMS Kokyuroko, 2015.

[39] K. Ueno, "Introduction to conformal field theory with gauge symmetries," in Geometry and Physics, ser. Proceedings of the Conference at Aarhus Univeristy. Aaarhus, Denmark: New York: Marcel Dekker, 1997.

[40] A. Yamada, "Precise variational formulas for abelian differentials," Kodai Math. J., vol. 3, pp. 114-143, 1988.

[41] Y. Zhu, "Modular invariance of characters of vertex operator algebras," J. Amer. Math. Soc., vol. 9, pp. 237-302, 1996.

[42] A. Zuevsky, "Identities for modular forms generated by vertex algebras," Miskolc Mathematical Notes, vol. 14, no. 2, pp. 721-727, 2013. 
[43] A. Zuevsky, "Twisted correlation functions on self-sewn Riemann surfaces via generalized vertex algebra of intertwiners," in Conformal Field Theory, Conformal Field Theory,Automorphic Forms and Related Topics, ser. Contributions in Mathematical and Computational Sciences, vol. 8. Berlin Heidelberg: Springer-Verlag, 2014.

[44] A. Zuevsky, "Frobenius-Fay identities for Jacobi forms," to appear, 2015.

[45] A. Zuevsky, "Towards automorphic to geometric correspondence for vertex algebras," in Bayesian Inference and Maximum Entropy Methods in Science and Engeineering, ser. MAXENT 2014, A. Mohammad-Djafari and F. Barbaresco, Eds., vol. 1641. Clos Luce, Amboise, France: AIP, 2015 .

Author's address

Alexander Zuevsky

Institute of Mathematics, Czech Academy of Sciences, Prague

E-mail address: zuevsky@yahoo.com 